\title{
Monte Carlo Simulation of Macromolecular Ionization by Nanoelectrospray
}

\author{
Christopher J. Hogan Jr. and Pratim Biswas \\ Aerosol and Air Quality Research Laboratory, Department of Energy, Environmental, and Chemical \\ Engineering, Washington University in St. Louis, St. Louis, Missouri, USA
}

\begin{abstract}
Electrospray ionization (ESI) is commonly used in macromolecular mass spectrometry, yet the dynamics of macromolecules in ESI droplets are not well understood. In this study, a Monte Carlo based model was developed, which can predict the efficiency of electrospray ionization for macromolecules, i.e., the number of macromolecular ions produced per macromolecules electrosprayed. The model takes into account ESI droplet evaporation, macromolecular diffusion within the droplet, droplet fissions, and the statistical nature of the ESI process. Two idealized representations of macromolecular analytes were developed, describing cluster prone, droplet surface inactive macromolecules and droplet surface active macromolecules, respectively. It was found that surface active macromolecules are preferentially ionized over surface inactive cluster prone macromolecules when the initial droplet size is large and the analyte concentration in solution is high. Simulations showed that ESI efficiency decreases with increasing initial droplet size and analyte molecular weight, and is influenced by analyte surface activity, the properties of the solvent, and the variance of the droplet size distribution. Model predictions are qualitatively supported by experimental measurements of macromolecular electrospray ionization made previously. Overall, this study demonstrates the potential capabilities of Monte Carlo based ESI models. Future developments in such models will allow for more accurate predictions of macromolecular ESI intensity. (J Am Soc Mass Spectrom 2008, 19, 1098-1107) (c) 2008 American Society for Mass Spectrometry
\end{abstract}

$\mathrm{E}$ lectrospray ionization (ESI) allows for the production of gas-phase ions from nonvolatile species in solution, and is particularly useful in the measurement of biological macromolecules with masses in the $\mathrm{kDa}$ and $\mathrm{MDa}$ ranges. Despite its widespread use, many aspects of the ESI of macromolecules are poorly understood [1]. The efficiency of the ESI process, i.e., the number of gas-phase ions produced per number of analytes electrosprayed, is a fundamental parameter in ESI that is typically unknown [2]. Droplets of the electrosprayed solution are produced in ESI, which have multiple excess charges on their surface [3]. Analytes are ionized from these droplets by one of two mechanisms: the charged residue mechanism [4] or the ion emission mechanism $[5,6]$. Analyte ionization by the charge residue mechanism requires that analytes be separated from one and other (one analyte per droplet) [7]. This is accomplished by Coulombic fissions of evaporating charged droplets in which unstable droplets emit smaller, charged progeny droplets [8]. Although complete separation of analytes is not necessary in the ion emission mechanism, ESI droplets must have diameters about $10 \mathrm{~nm}$ for ion emission to occur [6], and ion emission also occurs only after a series of droplet fission events [9].

Address reprint requests to C. J. Hogan Jr., Aerosol and Air Quality Research Laboratory, Department of Energy, Environmental, and Chemical Engineering, P.O. Box 1180, Washington University in St. Louis, St. Louis, MO 63130, USA. E-mail: cjhogan@wustl.edu
The parameters governing the ionization of small analytes, which are believed to be ionized by the ion emission mechanism [10], have been examined experimentally and theoretically $[9,11-18]$. In these studies, the efficiency of ionization was related to the chemical properties of the analytes in the droplet and their equilibrium partitioning between the inner and outer regions of ESI droplets. It was determined that the factor limiting the conversion of solution phase analytes to gas-phase ions was the availability of excess charge on ESI droplets. Conversely, experimental evidence suggests that ESI of macromolecules, with masses in the $\mathrm{kDa}$ range and larger, occurs by the charge residue mechanism $[7,19,20]$. While models based on equilibrium partitioning between inner and outer droplet may be sufficient for describing ESI of small, field emitting ions, an alternative model is needed to explain ESI of larger macromolecules. Macromolecules in aqueous solution can behave similar to colloidal particles, and their distribution and motion within an evaporating droplet is not only determined by their chemical properties but also the physical properties of the droplet and the macromolecules.

Few experimental studies have examined the parameters that determine the efficiency of the ESI process for macromolecules. McLuckey and coworkers [21] measured the signal intensities (related directly to the ionization efficiency) of analyte ions from protein mixtures electrosprayed at various $\mathrm{pH}$ values and deter- 
mined that the main parameter influencing ionization efficiency was the protein surface charge. Likewise, Kuprowski and Konermann [22] showed that the signal intensity of ions from ESI of denatured proteins with higher surface charge is greater than the signal intensity of ions from the ESI native state proteins. Thus far, however, a model of the ESI process for macromolecules has not been developed. The purpose of this work was to construct a Monte Carlo based model taking into account heat and mass transfer in ESI droplets, macromolecule diffusion in ESI droplets, and droplet fissions, which can be used to predict the efficiency of ESI for macromolecules. The model was used to determine the analyte ionization efficiency and signal intensity as a function of the ESI droplet size, analyte concentration in solution, analyte molecular weight, analyte surface activity, and variance in droplet size. Commonly used conditions for nanoelectrospray (nano-ESI) [23] were simulated. Simulated data were compared qualitatively to measurements of macromolecules by ESI-MS found in the literature.

\section{Theory and Model Development}

Macromolecular ESI was modeled accounting for droplet evaporation and Coulombic fissions. A physical representation of the model is shown in Figure 1. A similar model has been used to determine the structure of films formed by the deposition of electrosprayed nanoparticles [24] and the size distribution of nanoparticles produced by electrospray [25]. An initial diameter and excess surface charge for a single ESI droplet were specified, and the number of analytes in the droplet was randomly chosen from a Poisson distribution. The droplet then evaporated and analytes moved randomly within the droplet by Brownian motion, until the drop- let reached the Rayleigh limit. At the Rayleigh limit, the droplet underwent fission and lost mass and charge in the form of progeny droplets [8]. The number of analytes in each progeny droplet was selected from a Poisson distribution based on the analyte concentration in the outer region of the main droplet. Two analyte types were considered, cluster prone (CP) and surface active (SA). CP analytes formed ions if and only if there was one analyte per progeny droplet. SA analytes formed ions so long as the volume fraction of SA analytes in the progeny droplets was less than a critical volume fraction [26], taken as 0.50 for these calculations. After a fission event, the main droplet was then allowed to evaporate until Rayleigh limit was again reached. This procedure was repeated for multiple droplets to determine the electrospray ionization efficiency for a given solvent, droplet size, analyte molecular weight, analyte surface activity, and analyte concentration. Ionization efficiencies were calculated from the ratio of ionized analytes to total analytes electrosprayed. Details on the development of model initial conditions, droplet evaporation and analyte diffusion, droplet fission, analyte ionization, and efficiency calculation are given in the following sections. Readers not concerned with the technical details of model development may refer directly to the Results and Discussion section.

\section{ES Droplet Initial Conditions}

First, an ESI droplet diameter, $D_{D}$, was selected. The droplet contained a discrete number of charges, $n_{e}$, which were uniformly distributed on the droplet surface. The number of charges on the droplet surface was initially a fraction, $f_{r}$, of the number of charges at

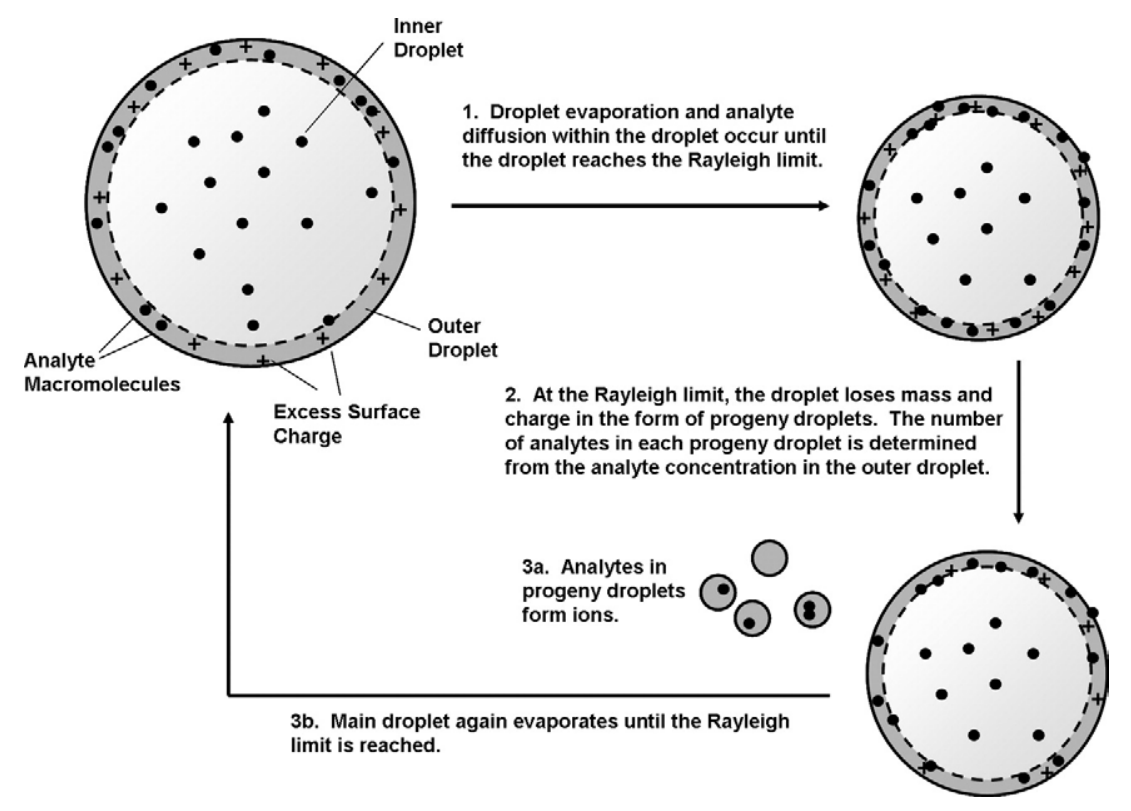

Figure 1. Representation of the ESI simulation. 
the Rayleigh limit [27], and was calculated from the equation:

$$
n_{e}=f_{R} Q_{R}=f_{R} \frac{\pi}{e}\left(8 \varepsilon_{0} \sigma D_{D}^{3}\right)^{1 / 2}
$$

where $Q_{R}$ is the number of charges on the droplet at the Rayleigh limit, $e$ is the unit electron charge $\left(1.6 \times 10^{-19}\right.$ C), $\varepsilon_{0}$ is the permittivity of a vacuum $\left(8.854 \times 10^{-12} \mathrm{C}^{2}\right.$ $\mathrm{N}^{-1} \mathrm{~m}^{-2}$ ), and $\sigma$ is the solvent surface tension. $f_{r}$ is a function of the dielectric constant of the electrosprayed solvent, and is estimated to be between 0.55 and 1.00 for most solvents [27-29]. For simulations in this study, a value of 0.70 was used for $f_{r}$, as has been used previously [8].

The ESI droplet contained a discrete number of analyte macromolecules. The average number of analytes per droplet, $N_{a v}$ was calculated as:

$$
N_{a v}=\frac{\pi}{6} C_{n u m} D_{D}^{3}
$$

where $C_{\text {num }}$ is the number concentration of analytes in solution. In ESI, the number of analytes in each droplet is Poisson distributed [24, 30,31]; thus, the number of analytes in the selected droplet, $N_{\text {anal }}$ was chosen randomly from the Poisson distribution function:

$$
P\left(N_{a n a l}\right)=\frac{e^{-N_{a v}} N_{a v}^{N_{a n a l}}}{N_{a n a l} !}
$$

where $P\left(N_{\text {anal }}\right)$ is the probability that a droplet that contained exactly $N_{\text {anal }}$ analytes. During the droplet formation process, it was assumed that analytes were distributed randomly within the droplet, regardless of the chemical nature of the analytes. Following calculation of the number of analytes, analytes were positioned at random locations within the droplet. Only droplets with more than one analyte were simulated, as droplets with only one analyte would always give rise to a gas-phase analyte ion. Single analyte droplets were later accounted for in efficiency calculations (see Efficiency Calculations section).

\section{Droplet Evaporation and Analyte Motion}

Once the initial droplet size, charge, and number of analytes were determined for a droplet, it was allowed to evaporate. The change in droplet diameter with time due to evaporation was calculated using the using the Fuchs-interpolation equation [32]:

$$
\frac{d D_{D}}{d t}=\frac{-4 \theta v_{m}}{D_{D} k T_{D}} g\left(D_{D}\right) p_{D}
$$

where $\theta$ is the diffusion coefficient of a gas-phase solvent molecule, $v_{m}$ is the volume of the evaporating solvent molecule, $k$ is $1.38 \times 10^{-23} \mathrm{~J} / \mathrm{K}, T_{D}$ is the droplet temperature, $p_{D}$ is the solvent partial pressure at the evaporating droplet surface, and $g\left(D_{D}\right)$ was calculated from the equation [32]:

$$
g\left(D_{D}\right)=\frac{1+2 \lambda / D_{D}}{1+3.42 \lambda / D_{D}+5.33\left(\lambda / D_{D}\right)^{2}}
$$

where $\lambda$ is the mean free path of the gas molecules surrounding the droplet. The partial pressure of the solvent was calculated using the Kelvin relation [32]:

$$
p_{D}=p_{s} e\left(\frac{4 \sigma v_{m}}{D_{D} k T_{D}}\right)
$$

where $p_{s}$ is the saturation pressure of the solvent. In ESI, heat transfer between the droplet and the surrounding bath gas occurs concurrently with droplet evaporation. Spatial variation of droplet temperature was neglected, and the change in droplet temperature over time was calculated from the equation [33]:

$$
\frac{d T_{D}}{d t}=\frac{12 K_{g}}{D_{D}^{2} \rho c_{p}}\left(T_{g}-T_{D}\right)+\frac{\pi H_{\text {vap }} \rho}{2} D_{D}^{2} \frac{d D_{D}}{d t}
$$

where $K_{g}$ is the thermal conductivity of the surrounding gas, $\rho$ is the solvent density, $c_{p}$ is the solvent specific heat, $T_{g}$ is the surrounding gas temperature and $H_{\text {vap }}$ is the solvent heat of vaporization. The differential equations in (eq 4a and eq 5) were solved in a coupled manner using a fourth order Runge-Kutta procedure to determine the change in droplet diameter over time. In all simulations, water was used as the solvent, for which the density is $1000 \mathrm{~kg} \mathrm{~m}^{-3}$, the specific heat is $1.88 \mathrm{~kJ} \mathrm{~kg}^{-1} \mathrm{~K}^{-1}$, the heat of vaporization is $2.26 \times 10^{6}$ $\mathrm{kJ} \mathrm{kg}^{-1}$, the surface tension is $0.073 \mathrm{~N} \mathrm{~m}^{-1}$, and the molecular volume is $2.99 \times 10^{-29} \mathrm{~m}^{3}$. Air was used as the surrounding gas, which has a thermal conductivity of $0.026 \mathrm{~W} \mathrm{~m}{ }^{-1} \mathrm{~K}^{-1}$. The saturation pressure of water, the vapor diffusion coefficient in air, and the mean free path of gas molecules vary with temperature; thus, experimentally-based regression equations [32, 34, 35] were used to calculate these parameters during the evaporation process.

While the droplet evaporated, the analyte macromolecules within the droplet moved randomly due to Brownian motion. Analyte macromolecules were modeled as colloidal particles, and their change in position over time was determined using the steady-state, inertia neglected, discrete form of the Langevin equation, given as [36, 37]:

$$
\begin{aligned}
& \vec{r}_{p, t+\Delta t}=\vec{r}_{p, t}+B \\
& \left\langle B^{2}\right\rangle=2 \frac{k_{b} T}{3 \pi \mu d_{a}} \Delta t
\end{aligned}
$$

where $\vec{r}$ is the analyte position vector, $B$ is a Gaussian random variable accounting for analyte Brownian mo- 
tion, $d_{a}$ is the analyte mobility diameter, $\mu$ is the dynamic viscosity of the solvent $t$ is time, and $\Delta t$ is the time step used for solving both equations (eqs 4, 5 and $6)$. Dynamic viscosities for water at varying temperatures were determined using interpolation with experimentally measured viscosity values [38]. The mobility diameter of a macromolecule of a given molecular weight was determined using curve fitting with experimentally measured mobility diameters of native state proteins [39, 40]. The motion of each analyte was monitored within the droplet, and the influence of any inter-analyte forces was neglected. While this assumption is reasonable for analytes in solvents with high electrical conductivity, as are commonly used in ESI, future implementations of this model may require the use of inter-analyte potential functions. Analyte-analyte collisions were also neglected. The motion of analytes was restricted at the surface of the droplet such that analytes could not escape the droplet by Brownian diffusion.

\section{Coulombic Fissions and Progeny Droplet Formation}

During evaporation, it was assumed that the charge on the droplet surface, $n_{e}$, was held constant. Evaporation of the selected droplet was allowed to proceed until the droplet reached its Rayleigh diameter, $D_{R}$, defined in terms of the number of charges on its surface:

$$
D_{R}=\left(\left(\frac{n_{e} e}{\pi}\right)^{2} \frac{1}{\sigma \varepsilon_{0}}\right)^{1 / 3}
$$

At the Rayleigh diameter, the droplet underwent fission and lost mass and charge in the form of smaller progeny droplets. A fission event can be described by three parameters [8]: the droplet mass loss during the fission, $l_{m-}$, the droplet charge loss during the fission, $l_{c}$, and the diameter of the progeny droplets, $D_{p D}$. During the fission event, the diameter of the droplet and charge on the droplet were reduced according to the equations:

$$
\begin{aligned}
& D_{D}=\left(1-l_{m}\right)^{1 / 3} D_{R} \\
& n_{e}=\left(1-l_{c}\right) n_{e, p}
\end{aligned}
$$

where $n_{e, p}$ is the charge on the droplet before the fission. Several experimental studies have measured the mass and charge loss in droplet fissions [41, 42]. However, measurements were made on droplets with diameters $>1 \mu \mathrm{m}$, which are much larger than the droplets produced in nano-ESI [43]. In this study, varying values of $l_{m}$ and $l_{c}$ were used to determine their affects on the ionization process. When other model parameters were varied, fixed values of $l_{m}=0.02$ and $l_{c}=0.15$ were used, which are in the range of experimental measurements and were also used by Tang and Smith [3] as well as Cech and Enke [9] in modeling droplet fissions.
Fernandez de la Mora [8] has developed a model that can be used to calculate the diameter of progeny droplets. However, this model generally applies only to droplets with diameters about micrometers. Based on the observations of Chen et al. [43] of nano-sized electrospray droplet diameters, the progeny droplet diameters in all simulations were calculated from the equation:

$$
D_{p D}=\frac{D_{R}}{8}
$$

Once $l_{m}, l_{c}$, and $D_{p D}$ were specified, the number of progeny droplets produced during a fission event, $N_{p D}$, was calculated from the equation:

$$
N_{p D}=l_{m}\left(\frac{D_{R}}{D_{p D}}\right)^{3}
$$

The fission event in which progeny droplets form is similar to the formation of the original droplets in ESI [44]. Therefore, the number of analyte macromolecules in progeny droplets would follow a Poisson distribution. However, not all of the droplet volume can be ejected during a fission event; the mass and charge lost during a fission event come from the outer region of the droplet [44]. For the purpose of modeling droplet fissions, the droplet was divided into inner and outer regions, as has been suggested by Enke and coworkers $[9,13]$. The inner to outer droplet volume ratio was taken as 4:1 in this study. No measurements have been made previously of the inner to outer droplet volume ratio; thus, the value used in this study was a first approximation. The droplet mass in the outer region was accessible to the fission; i.e., progeny droplets formed from the outer droplet region. At the time of the fission event $\left(D_{D}=D_{R}\right)$, the concentration of analytes in the outer droplet region was calculated. Using the concentration of analytes in the outer droplet region and the diameter of the progeny droplets, the number of analytes in each progeny droplet formed was calculated randomly from a Poisson distribution function (eq 3 ). The analytes found in progeny droplets were removed from the outer region of the original droplet. Droplet evaporation was then allowed to continue until the droplet again reached the Rayleigh size and it subsequently underwent fission. The evaporation/ fission process proceeded until either all analytes escaped the droplet or until the volume fraction of solvent in the droplet decreased below a critical volume fraction, at which point macromolecular entanglements formed within the droplet, and fission could no longer occur [26]. For these simulations, a modest critical volume fraction of 0.50 was used (fission preventing entanglements can form in many macromolecular solutions at higher solvent volume fractions than 0.50). If a single analyte remained in the parent droplet at the time when solvent evaporation was complete, it formed a gas-phase analyte ion. 


\section{Analyte Ion Formation from Progeny Droplets}

Gas-phase analyte ions could form from the analytes in progeny droplets [3]. It was assumed that all analyte macromolecules were ionized by the charge residue mechanism and, therefore, analyte separation during the progeny droplet evaporation/fission process was required for analyte ionization. However, tracking the evaporation and fission of all progeny droplets formed from all original droplets would be too computationally intense for a practical model, as a single ESI droplet will give rise to a multitude of progeny droplets during the evaporation/fission process. As an alternative, analyte ionization from progeny droplets was determined by whether analytes were one of two theoretical analyte types: cluster prone surface inactive $(\mathrm{CP})$ or surface active (SA) [22]. CP analytes were modeled as particles that would cluster in progeny droplets and did not form gas-phase ions unless there was only one analyte in a progeny droplet. Progeny droplets that contained multiple $\mathrm{CP}$ analyte macromolecules in this model evaporated and left residual macromolecular $n$-mers, which would not contribute to analyte signal [2]. For the SA analyte model, it was assumed that SA analytes would not cluster in progeny droplets, and therefore all SA analytes formed gas-phase ions unless the solvent volume fraction in the progeny droplets was less than 0.50. Isolated macromolecules in both models were able to hold excess charge and become ionized in the gas phase. Furthermore, it was assumed that small molecule ion emission, which can occur from macromoleculecontaining nanodroplets [7], did not completely deplete the droplet of charge before complete solvent evaporation.

\section{Efficiency Calculations}

After the evaporation-fission process completed for a droplet, the number of ions that were produced from both CP and SA analytes was recorded, and the evaporation-fission process was carried out for a new droplet. Simulations were repeated until 100 droplets, and enough droplets to contain 5000 analytes were simulated (both criteria needed to be met). Simulations were only performed on droplets that contained more than one analyte. Using simulated data, the ionization efficiency under specific ESI conditions, IE, was calculated as:

$$
I E=\frac{P(1)}{1-P(0)}+\frac{1-P(1)-P(0)}{1-P(0)} \frac{\sum_{l=1}^{l=m} I_{l}}{\sum_{l=1}^{l=m} N_{\text {Anal }, l}}
$$

where $m$ is the number of droplets simulated under a given set of conditions, $I_{l}$ is the number of analyte ions

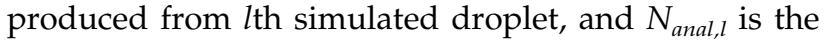
total number of analytes originally contained within the $l$ th simulated droplet. In eq 11, the first term represents statistically-based ionization, i.e., the droplets that contain only one analyte, while the second term represents ionization due to droplet fissions. As $I_{l}$ differed for $\mathrm{CP}$ and SA analytes, a CP ionization efficiency and SA ionization efficiency were calculated for each simulation performed.

\section{Results and Discussion}

The effects of a variety of ESI operational parameters on the ionization efficiency were investigated in this study. A summary of the test cases modeled and parameter values used in simulations is given in Table 1 . The effects of initial ESI droplet size, analyte molecular weight, and charge and mass loss during fissions events were studied systematically.

\section{Initial Droplet Size}

The ionization efficiency (eq 11) of CP and SA $20 \mathrm{kDa}$ analytes from ESI droplets with initial droplets of 100 $\mathrm{nm}, 200 \mathrm{~nm}$, and $300 \mathrm{~nm}$ are shown in Figure 2a as a function of analyte concentration. Error bars represent the standard deviation of the ionization efficiency between individual droplets. At low analyte concentrations, regardless of initial droplet size or analyte type, the ionization efficiency was close to 1.0, indicating that under these conditions, all droplets either contained a single analyte or analytes were successfully separated from each other by Coulombic fissions. The ionization efficiency decreased below 1.0 at higher analyte concen-

Table 1. Summary parameters used in ESI simulations

\begin{tabular}{|c|c|}
\hline Varied parameter & Parameter values \\
\hline $\begin{array}{l}\text { Initial droplet diameter } \\
(100 \mathrm{~nm}, 200 \mathrm{~nm}, \\
300 \mathrm{~nm})\end{array}$ & $\begin{array}{l}\text { Explosion mass loss, } I_{m}: 0.02 \\
\text { Explosion charge loss, } I_{c}: 0.15 \\
\text { Analyte concentration: } 0.1-1000 \mu \mathrm{M} \\
\text { Bath gas temperature, } T_{g}: 100^{\circ} \mathrm{C} \\
\text { Analyte molecular weight: } 20 \mathrm{kDa}^{\text {a }}\end{array}$ \\
\hline $\begin{array}{l}\text { Analyte molecular } \\
\text { weight }(2 \mathrm{kDa}, 20 \\
\mathrm{kDa}, 200 \mathrm{kDa})^{\mathrm{a}}\end{array}$ & $\begin{array}{l}\text { Explosion mass loss, } I_{m}: 0.02 \\
\text { Explosion charge loss, } I_{c}: 0.15 \\
\text { Analyte concentration: } 0.1-1000 \mu \mathrm{M} \\
\text { Bath gas temperature, } T_{g}: 100^{\circ} \mathrm{C} \\
\text { Initial droplet diameter: } 200 \mathrm{~nm}\end{array}$ \\
\hline $\begin{array}{l}\text { Explosion mass loss, } \\
\qquad I_{m}: 0.01-0.05\end{array}$ & Analyte concentration: $30 \mu \mathrm{M}$ \\
\hline $\begin{array}{l}\text { Explosion charge loss, } \\
I_{c}: 0.10-0.20\end{array}$ & $\begin{array}{l}\text { Bath gas temperature, } T_{g}: 100^{\circ} \mathrm{C} \\
\text { Analyte molecular weight: } 20 \mathrm{kDa}^{\mathrm{a}} \\
\text { Initial droplet diameter: } 200 \mathrm{~nm}\end{array}$ \\
\hline $\begin{array}{l}\text { Geometric standard } \\
\text { deviation of initial } \\
\text { droplet }\end{array}$ & Explosion mass loss, $I_{m}: 0.02$ \\
\hline $\begin{array}{l}\text { Size distribution, } \sigma_{\mathrm{g}} \\
\quad(1.0,1.2,1.6,2.0)\end{array}$ & $\begin{array}{l}\text { Explosion charge loss, } I_{c}: 0.15 \\
\text { Analyte concentration: } 0.1-1000 \mu \mathrm{M} \\
\text { Bath gas temperature, } T_{g}: 100^{\circ} \mathrm{C} \\
\text { Analyte molecular weight: } 20 \mathrm{kDa}^{\mathrm{a}} \\
\text { Initial Droplet Diameter: } 200 \mathrm{~nm}^{\mathrm{b}}\end{array}$ \\
\hline
\end{tabular}

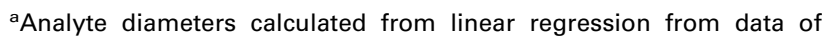
Kaddis et al. [40]; $2 \mathrm{kDa}-2.2 \mathrm{~nm}, 20 \mathrm{kDa}-4.8 \mathrm{~nm}, 200 \mathrm{kDa}-10.3 \mathrm{~nm}$. ${ }^{b}$ Geometric mean initial droplet diameter. 


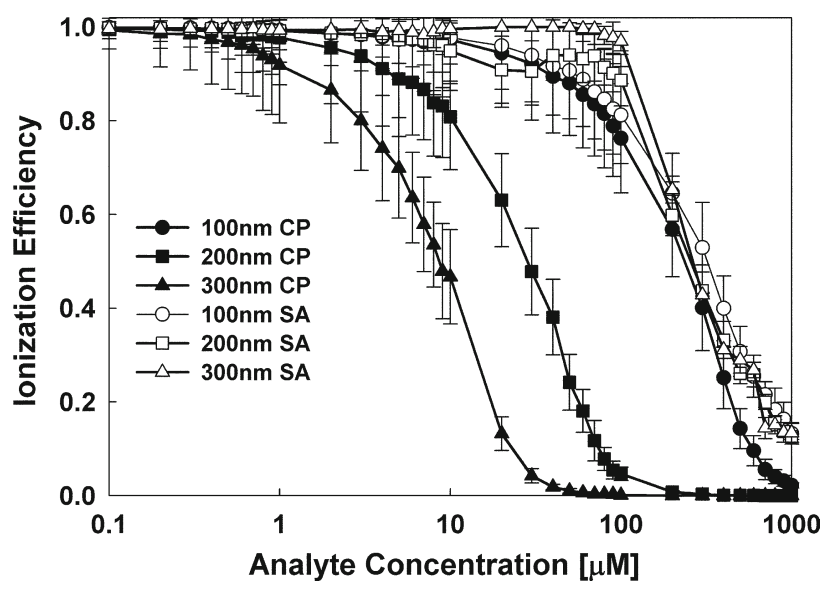

Figure 2. Efficiency of $20 \mathrm{kDa}$ macromolecule gas-phase ion formation as a function of macromolecular concentration in solution

trations. There was a sharp difference in the behavior of $\mathrm{CP}$ and SA analytes. The ionization efficiency was strongly a function of initial droplet diameter for $\mathrm{CP}$ analytes, with larger ESI droplets having lower ionization efficiencies. This agrees well with several experimental studies [14, 45] where ESI efficiency was found to increase with decreasing ESI flow rates (lower flow rates generally result in the production of smaller droplets). In this model, ionization efficiency decreased due to the formation of macromolecular $n$-mers, i.e., the analytes were not completely separated from each other during the evaporation-fission process. The ionization efficiency was also plotted as a function of the average number of analytes per droplet (see Supporting Information, which can be found in the electronic version of this article). For CP analytes, the ionization efficiency as a function of the average number of analytes per droplet was independent of initial droplet diameter, indicating that $\mathrm{CP}$ analyte ionization is a statistical phenomenon, dependent on the number of analytes in the outer region of the droplet at the time of fission. Conversely, for SA ions, ionization efficiency versus analyte concentration curves was near-identical to each other (Figure 2). Ionization efficiency in the SA ionization model was also dependant the formation of $n$-mers, however, the $n$-mer formation occurred at a critical progeny droplet volume fraction, and therefore droplet size had no effect on the ionization process.

In addition to the ionization efficiency, the fraction of analytes derived from each fission, e.g., one-tenth of the ions may come from the fourth fission, is also of interest [9]. The fraction of $\mathrm{CP}$ and $\mathrm{SA}$ analytes originating from the first 25 droplet fissions are shown in Figure $3 \mathrm{a}$ and $\mathrm{b}$, respectively. For both ion types, most analyte ions were produced after the first fission, indicating that droplet evaporation must complete to maximize analyte ionization. This has implications on techniques such as charge reduction ESI [46], in which model results show it is preferable to charge reduce analytes after droplet evaporation, as charge reduction prevents droplet fis- sions from occurring. In instances of high analyte concentration $(100 \mu \mathrm{M})$, analyte ionization primarily occurred during the first few fissions. During the evaporation process, the analyte concentration in ESI droplets produced from concentration solutions became sufficiently high in the outer droplet region; thus, any progeny droplets produced contained too many analytes to be separated by later droplet fissions.

\section{Analyte Molecular Weight and Surface Activity}

Large analytes diffuse slower than small analytes in an evaporating droplet, and for a given analyte molarity, they make up a larger volume fraction of droplets. Therefore, analyte molecular weight, i.e., physical size of the analyte, affects ESI efficiency. The ESI efficiency as a function of concentration for a $200 \mathrm{~nm}$ droplet with analyte molecular weights of 2,20 , and $200 \mathrm{kDa}$ is shown in Figure 4a. As was found with droplet size, the effect of analyte molecular weight on the ionization efficiency of CP analytes differed from its affect on SA analytes. The ionization efficiency of $\mathrm{CP}$ analytes was not influenced by analyte molecular weight because the clustering and decrease in ionization efficiency of $\mathrm{CP}$ analytes was due to increases in number concentration
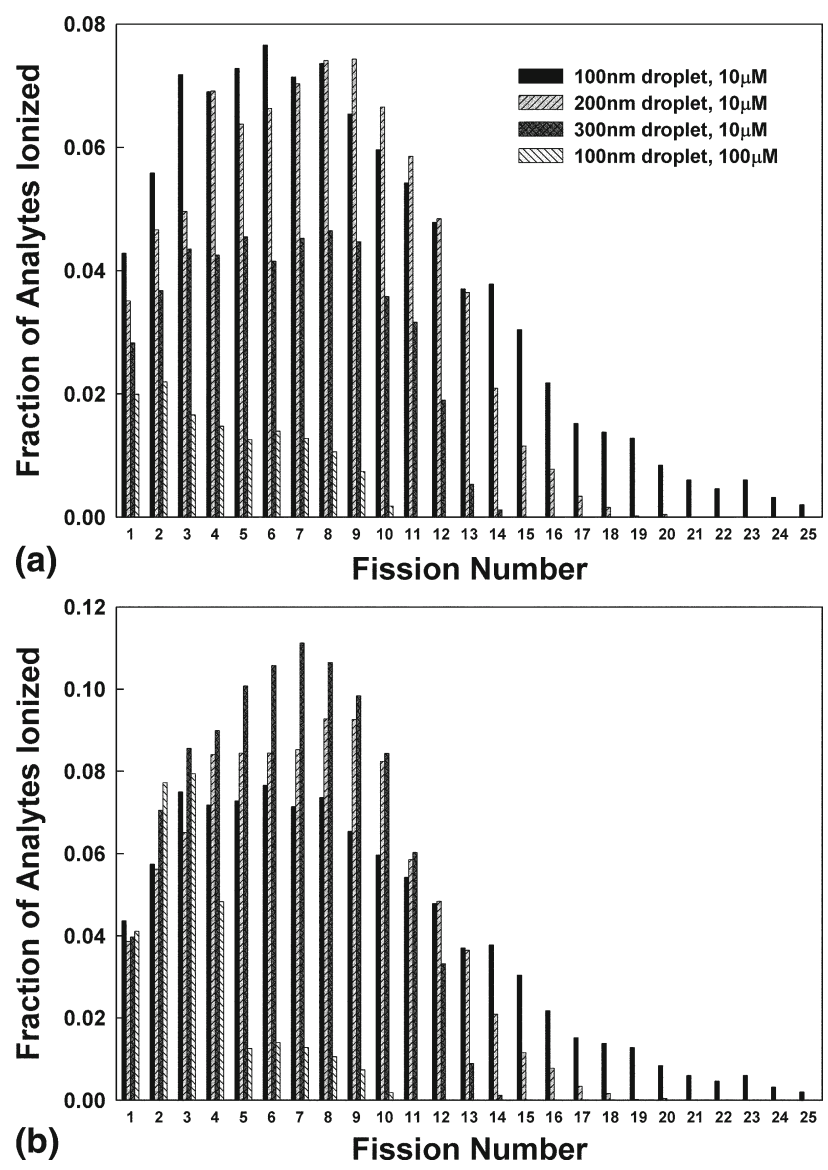

Figure 3. The fraction of (a) cluster prone and (b) surface active macromolecules ionized in each successive fission of the parent droplet. 

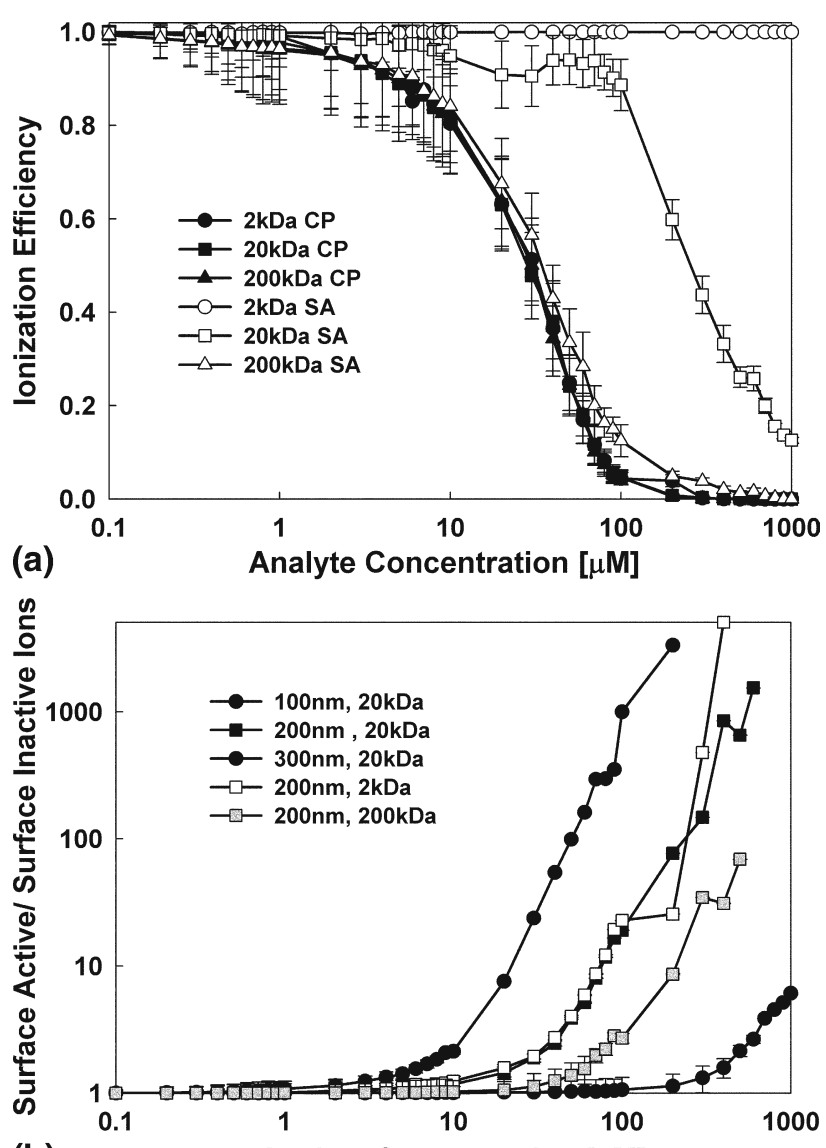

(b) Analyte Concentration $[\mu \mathrm{M}]$

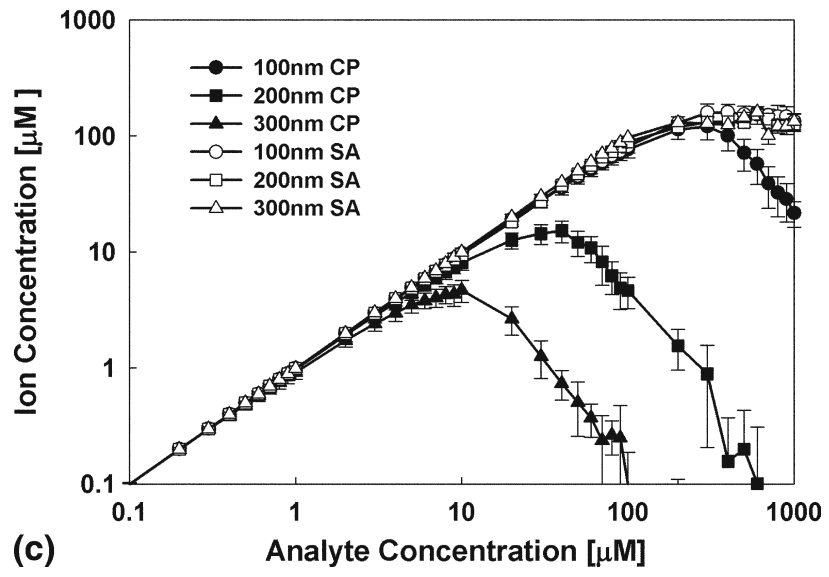

Figure 4. (a) Efficiency of macromolecular gas-phase ion formation as a function of macromolecular concentration in solution for different molecular weight analytes. (b) Ratio of surface active ions to cluster prone ions produced as a function of analyte concentration for different droplet sizes and analyte molecular weights. (c) Relative analyte ion concentration in solution as a function of analyte concentration for $20 \mathrm{kDa}$ analytes.

in the outer region of the fissioning droplet. Higher molecular weight SA analytes, however, made up a greater volume fraction in progeny droplets compared with lower molecular weight analytes and, therefore, did not ionize as efficiently as low molecular weight analytes.

In all simulations with analytes of different molecular weight and different initial droplet sizes, SA ana- lytes had higher ionization efficiencies than $\mathrm{CP}$ analytes. Figure $4 \mathrm{~b}$ shows the ratio of the ionization efficiency of SA analytes to the ionization efficiency of $\mathrm{CP}$ analytes for three initial droplet sizes and molecular weights. Simulations clearly showed that the ratio of SA ions to $\mathrm{CP}$ ions increased as analyte molarity increased, droplet size increased, and analyte molecular weight increased. Therefore, in the ionization of macromolecular mixtures of $\mathrm{CP}$ and SA analytes, SA analytes would be preferentially ionized from larger droplets, at higher analyte concentrations, or for smaller analytes. This modeled result is in good agreement with recent experimental studies of protein ESI. Although the models of $\mathrm{CP}$ and SA analytes used in this are study highly idealized in their depiction of analyte behavior, they describe the behavior of native state proteins and denatured proteins reasonably well. Native state proteins often have low net surface charge and would be less concentrated in the outer regions of ESI droplets [21, 47].

Therefore, native state proteins would be prone to clustering, and their behavior in evaporating and fissioning droplets would be similar to the modeled behavior of CP analytes. Denatured proteins often have high net surface charge, would be less likely to cluster (electrostatic repulsion), and would escape droplets during fissions events. Their behavior would be similar to the modeled behavior of SA analytes. It is not surprising that in a recent study by Kuprowski and Konermann [22], it was found that denatured proteins have higher measured signal intensities than do native state proteins, and the maximum difference in signal intensity between denatured and native state proteins occurred when higher ESI flow rates (larger initial droplets) and higher analyte concentrations were used. The difference in ion concentration was found to be as high as two orders of magnitude, which can be expected based upon the simulations performed here.

Simulations were also used to predict the analyte molarity at which the analyte ion signal intensity would be maximized (for a given initial droplet diameter). The effective analyte ion concentration (the product of the analyte molarity and the ionization efficiency) as a function of analyte concentration for $20 \mathrm{kDa}$ analytes with initial droplet diameters of 100, 200, and $300 \mathrm{~nm}$ is shown in Figure 4c. For both CP and SA analytes, at low analyte concentration, the effective analyte ion concentration was linearly proportional to the analyte concentration, as the ionization efficiency was 1.0 for both analyte types. $\mathrm{CP}$ ions reached a maximum value at an analyte concentration that was decreasing function of initial droplet diameter, i.e., the model showed that using nano-ESI rather than traditional ESI increases the effective analyte ion concentration of the solution. The SA effective ion concentration was unaffected by droplet diameter, as was expected based on the model results shown in Figure 2. SA effective ion concentrations (which relate directly to signal intensity), reached a maximum value and remained at the maximum value 
as analyte concentration was increased. This was also found experimentally by McLuckey and coworkers [21] for three different denatured (surface active) proteins.

Although the results of Pan and McLuckey [47] as well as Kuprowski and Konermann [22] are in good qualitative agreement with predictions from the model used here, the explanation of results used in those studies differed from the justifications of results based on this model. In both referenced studies, the observed results were attributed to the lack of available excess droplet surface charge to sufficiently charge all analytes. In the model used here, decreases in ionization efficiency are attributed to analyte clustering. Certainly, both phenomena, charge depletion and analyte clustering, can give rise to decreases in ESI efficiency and must be taken into consideration. For the 100, 200, and 300 $\mathrm{nm}$ droplets used in this study, the charge concentration ( $n_{e}$ in eq 1 divided by the droplet volume, given in moles of excess charge) in the initial droplet was 3127, 1106 , and $602 \mu \mathrm{M}$ respectively. For macromolecules and with nano-ESI, as were studied here, the effects of analyte clustering for both CP and SA analytes appear to be the limiting factor in ESI ionization, as efficiency decreases occurred in simulations for CP analyte concentrations around $10 \mu \mathrm{M}$ and SA analyte concentrations around $100 \mu \mathrm{M}$. Although macromolecules would presumably be multiply charged [7], analyte clustering would likely be the factor limiting complete analyte ionization at these concentrations. However, for small, surface active analytes, such as the $2 \mathrm{kDa}$ analyte simulated here, charge depletion would be limiting [48]. The influence of charge depletion on ESI efficiency would also be magnified if larger droplets (ESI rather than nano-ESI) were used. Future modeling efforts will need to take into account both analyte clustering and charge depletion, the presence of SA and CP ions in the same droplets (true modeling of macromolecular mixture ESI), and the effects of the presence of small cations $[7,48]$ to fully describe the limiting factor in ESI.

\section{Solvent Properties}

The mass and charge lost during fission events vary with solvent composition. The ionization efficiency of $20 \mathrm{kDa}$ CP and SA analytes from $200 \mathrm{~nm}$ droplets with an analyte concentration of $25 \mu \mathrm{M}$ are shown in Figure $5 \mathrm{a}$ and $\mathrm{b}$, respectively. For both analyte types, ionization efficiency increased with increasing mass loss during fission and with decreasing charge loss during fission. These results clearly show that solvent properties strongly influence ionization efficiency. Unfortunately, little work has been done on measurement of the mass and charge lost from nanometer sized droplets, but it will be necessary to do so in the future, particularly for solvents relevant to mass spectrometry, to fully understand ESI.
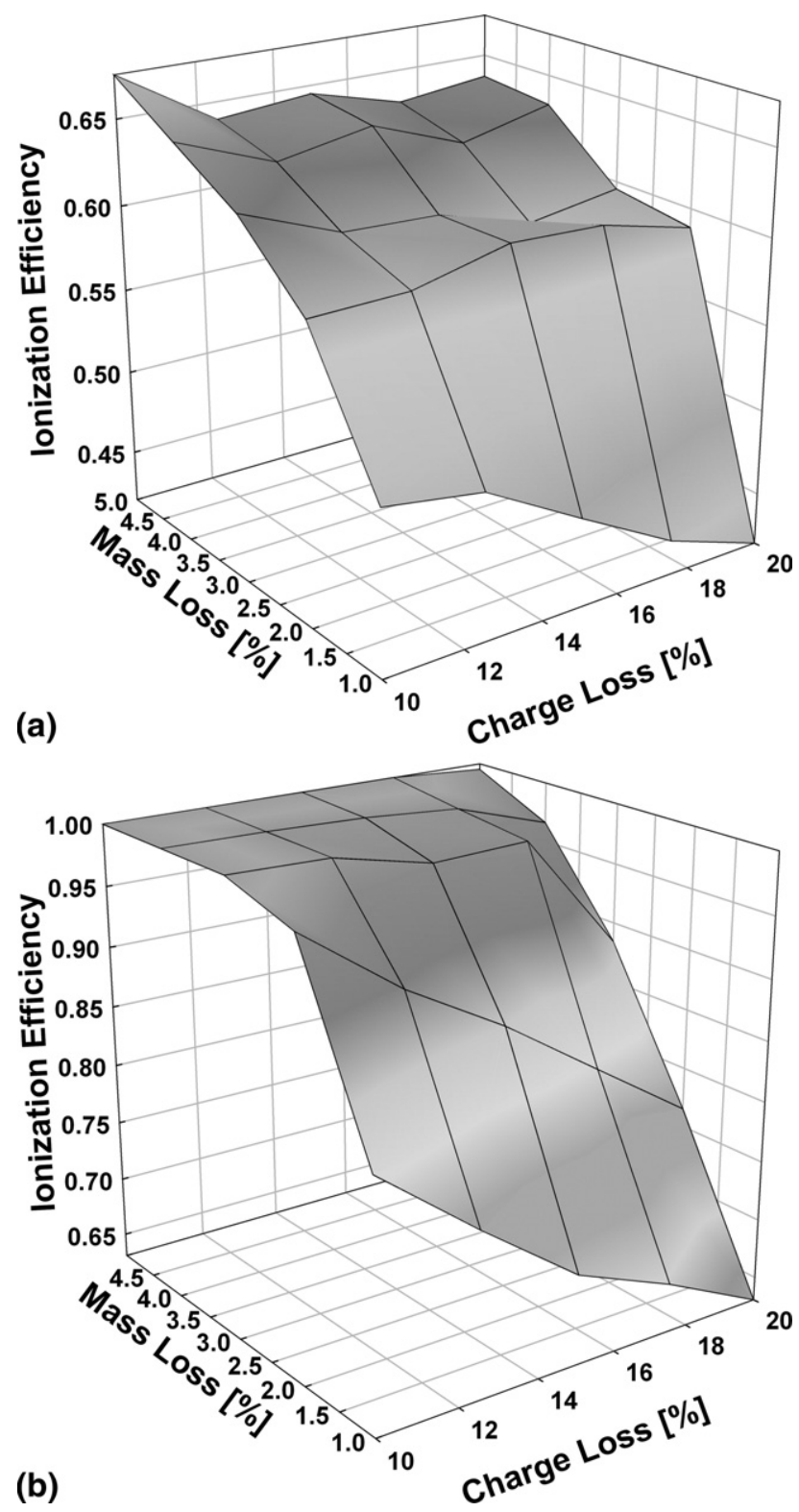

Figure 5. Ionization efficiency of (a) cluster prone and (b) surface active macromolecules as a function of the percent mass loss and percent charge loss of the parent droplet during each fission event.

\section{Initial Droplet Size Distribution}

In ESI-MS experiments, initial droplets do not all have the same diameter; rather, there is an initial droplet size distribution function, $\eta\left(D_{D}\right)$. Experimental studies [26, 30] of the diameter of initial droplets have shown that the size distribution function of ESI droplets can be described by the equation [24]:

$$
\eta\left(D_{D}\right)=\frac{1}{D_{D} \ln \left(\sigma_{g}\right) \sqrt{2 \pi}} \exp \left(\frac{-\left(\ln \left(D_{D}\right)-\ln \left(D_{D_{g}}\right)\right)^{2}}{2 \ln ^{2}\left(\sigma_{g}\right)}\right)
$$

where $D_{D}$ is the initial droplet diameter, $D_{D g}$ is the initial droplet geometric mean diameter (median diam- 


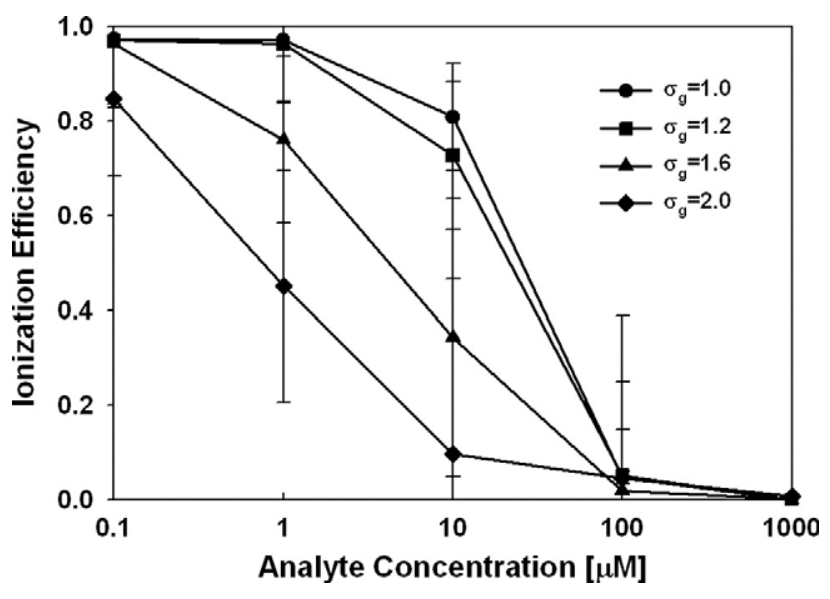

Figure 6. Ionization efficiency for $20 \mathrm{kDa} \mathrm{CP}$ macromolecules with different droplet size distribution geometric standard deviations. The geometric mean initial droplet diameter was $200 \mathrm{~nm}$.

eter), and $\sigma_{g}$ is the initial droplet size distribution geometric standard deviation. $\sigma_{g}$ in ESI systems varies with ESI spray mode, and can be as low as 1.05 in the cone-jet mode [26] and upwards of 2.0 for unstable ESI operation. Nemes et al. [49] recently studied the effect of spray mode on the ESI process experimentally and determined that signal intensities and, therefore, ESI efficiency was highest in the cone-jet mode, i.e., when the geometric standard was low.

The effect of initial droplet size distribution function on ESI efficiency was also determined here. Analyte ionization was simulated from a distribution with a specified $D_{D g}$ and $\sigma_{g}$ by first randomly selecting a droplet diameter from the distribution described by eq 13 [24], then carrying out the evaporation/fission process. This simulation procedure was repeated for 5000 droplets to sample the entire size distribution function. For simplicity in calculation, only the ionization due to the evaporation/fission process (second term in eq 11) was considered. The fission based ionization efficiency as a function of analyte concentration for $20 \mathrm{kDa} C P$ analytes is shown in Figure 6 for different values of $\sigma_{g}$. In all cases, the geometric mean droplet diameter was $200 \mathrm{~nm}$. With increasing geometric standard deviation, the ionization efficiency decreased at low concentrations. Size distribution functions with high geometric standard deviations describe droplet distributions that contain large droplets. These large droplets contain the majority of the analytes and have low ionization efficiencies. At high analyte concentration, the ionization efficiency was low regardless of the geometric standard deviation. The simulated results are in excellent agreement with the study of Nemes et al. [49], and as was cautioned in their work, spray mode has large effects on the ESI process.

\section{Conclusions}

The efficiency of nano-ESI for macromolecules was studied by developing a computational model, which considered the physics and statistics of ESI. This differs from previous models of ESI, which were developed for small analytes and relate the decrease in ESI efficiency to charge depletion. Precise knowledge of solvent properties, analyte properties, and droplet size are required for model predictions of ESI efficiency. In this sense, the current model is a basic skeleton for future modeling efforts in ESI. Refinements to the model based on subsequent experimental measurements of ESI solvent and analyte properties (e.g., interanalyte forces, estimates of the inner droplet-outer droplet partition ratio) should eventually allow for precise and accurate predictions for ESI-MS experiments, which will be invaluable in macromolecular analysis.

\section{Acknowledgments}

$\mathrm{CJH}$ acknowledges support from an NSF Graduate Research Fellowship. Partial support from a DOD-MURI grant is also acknowledged.

\section{References}

1. Constantopoulos, T. L.; Jackson, G. S.; Enke, C. G. Challenges in Achieving a Fundamental Model for ESI. Anal. Chim. Acta 2000, 406, 37-52.

2. Cech, N. B.; Enke, C. G. Practical Implications of Some Recent Studies in Electrospray Ionization Fundamentals. Mass Spectrom. Rev. 2001, 20, 362-387.

3. Tang, K. Q.; Smith, R. D. Theoretical Prediction of Charged Droplet Evaporation and Fission in Electrospray Ionization. Int. J. Mass Spectrom. 1999, 187, 97-105.

4. Dole, M.; Mack, L. L.; Hines, R. L.; Mobley, R. C.; Ferguson, L. D.; Alice, M. B. Molecular Beams of Macroions. J. Chem. Phys. 1968, 49, 2240-2249.

5. Gamero-Castano, M.; Fernandez de la Mora, J. Direct Measurement of Ion Evaporation Kinetics from Electrified Liquid Surfaces. J. Chem. Phys. 2000, 113, 815-832.

6. Iribarne, J. V.; Thomson, B. A. On the Evaporation of Small Ions from Charged Droplets. J. Chem. Phys. 1976, 64, 2287-2294.

7. Hogan, C. J.; Carroll, J. A.; Rohrs, H. W.; Biswas, P.; Gross, M. L. Charge Carrier Field Emission Determines the Number of Charges on Native States Proteins in Electrospray Ionization. J. Am. Chem. Soc. 2008, 130, 6926-6927.

8. Fernandez de la Mora, J. On the Outcome of the Coulombic Fission of a Charged Isolated Drop. J. Colloid Interface Sci. 1996, 178, 209-218.

9. Cech, N. B.; Enke, C. G. Effect of Affinity for Droplet Surfaces on the Fraction of Analyte Molecules Charged During Electrospray Droplet Fission. Anal. Chem. 2001, 73, 4632-4639.

10. Nguyen, S.; Fenn, J. B. Gas-Phase Ions of Solute Species from Charged Droplets of Solutions. Proc. Natl. Acad. Sci. U.S.A. 2007, 104, 1111-1117.

11. Tang, K.; Page, J. S.; Smith, R. D. Charge Competition and the Linear Dynamic Range of Detection in Electrospray Ionization Mass Spectrometry. J. Am. Soc. Mass Spectrom. 2004, 15, 1416-1423.

12. Cech, N. B.; Enke, C. G. Relating Electrospray Ionization Response to Nonpolar Character of Small Peptides. Anal. Chem. 2000, 72, 2717-2723.

13. Enke, C. G. A Predictive Model for Matrix and Analyte Effects in Electrospray Ionization of Singly-Charged Ionic Analytes. Anal. Chem. 1997, 69, 4885-4893.

14. El-Faramawy, A.; Siu, K. W. M.; Thomson, B. A. Efficiency of NanoElectrospray Ionization. J. Am. Soc. Mass Spectrom. 2005, 16, 1702-1707.

15. Bokman, C. F.; Bylund, D.; Markides, K. E.; Sjoberg, P. J. R. Relating Chromatographic Retention and Electrophoretic Mobility to the Ion Distribution Within Electrosprayed Droplets. J. Am. Soc. Mass Spectrom. 2006, 17, 318-324.

16. Zhou, S.; Cook, K. D. A Mechanistic Study of Electrospray Mass Spectrometry: Charge Gradients within Electrospray Droplets and Their Influence on Ion Response. J. Am. Soc. Mass Spectrom. 2001, 12, 206-214.

17. Sherman, C. L.; Brodbelt, J. S. Partitioning Model for Competitive Host-Guest Complexation in ESI-MS. Anal. Chem. 2005, 77, 2512-2523.

18. Haddrell, A. E.; Agnes, G. R. Organic Cation Distributions in the Residues of Levitated Droplets with Net Charge: Validity of the Partition Theory for Droplets Produced by an Electrospray. Anal. Chem. 2004, 76, 53-61.

19. Felitsyn, N.; Peschke, M.; Kebarle, P. Origin and Number of Charges Observed on Multiply-Protonated Native Proteins Produced by ESI. Int J. Mass Spectrom. 2002, 219, 39-62. 
20. Fernandez de la Mora, J. Electrospray Ionization of Large Multiply Charged Species Proceeds Via Dole's Charged Residue Mechanism. Anal. Chim. Acta 2000, 406, 93-104.

21. Pan, P.; Gunawardena, H. P.; Xia, Y.; McLuckey, S. A. Nanoelectrospray Ionization of Protein Mixtures: Solution pH and Protein pI. Anal. Chem. 2004, 2004, 1165-1174.

22. Kuprowski, M. C.; Konermann, L. Signal Response of Coexisting Protein Conformers in Electrospray Mass Spectrometry. Anal. Chem. 2007, 79, 2499-2506

23. Juraschek, R.; Dulcks, T.; Karas, M. Nanoelectrospray-More than Just a Minimized-Flow Electrospray Ionization Source. J. Am. Soc. Mass Spectrom. 1999, 10, 300-308.

24. Hogan, C. J.; Biswas, P. Porous Film Deposition by Electrohydrodynamic Atomization of Nanoparticle Sols. Aerosol Sci. Tech. 2008, 42, $75-85$.

25. Hogan, C. J.; Biswas, P. Narrow Size Distribution Nanoparticle Production by Electrospray Processing of Ferritin. J. Aeros. Sci. 2008, 39, $432-440$.

26. Hogan, C. J.; Yun, K.-M.; Chen, D. R.; Lenggoro, I. W.; Biswas, P.; Okuyama, K.; Controlled Size. Polymer Particle Production via Electrohydrodynamic Atomization. Colloids Surf. A Physicochem. Eng. Aspects 2007, 311, 67-76.

27. de Juan, L.; Fernandez de la Mora, J. Charge and Size Distributions of Electrospray Drops. J. Colloid Interface Sci. 1997, 186, 280-293.

28. Ganan-Calvo, A. M.; Barrero, A. Current and Droplet Size in the Electrospraying of Liquids Scaling Laws. J. Aerosol Sci. 1997, 28, 249275.

29. Chen, D. R.; Pui, D. Y. H. Experimental Investigation of Scaling Laws for Electrospraying: Dielectric Constant Effect. Aerosol Sci. Technol. 1997, 27, 367-380.

30. Hogan, C. J.; Kettleson, E. M.; Ramaswami, B.; Chen, D. R.; Biswas, P. Charge Reduced Electrospray Size Spectrometry of Mega- and Gigada1ton Complexes: Whole Viruses and Virus Fragments. Anal. Chem. 2006, $78,844-852$

31. Lewis, K. C.; Dohmeier, D. M.; Jorgenson, J. W.; Kaufman, S. L.; Zarrin, F.; Dorman, F. D. Electrospray-Condensation Particle Counter-a Molecule-Counting LC-Detector for Macromolecules. Anal. Chem. 1994, 66, 2285-2292.

32. Friedlander, S. K. Smoke, Dust, and Haze; Oxford University Press: New York, 2000; p. 285.

33. Lenggoro, I. W.; Hata, T.; Iskandar, F.; Lunden, M. M.; Okuyama, K. An Experimental and Modeling Investigation of Particle Production by Spray Pyrolysis Using a Laminar Flow Aerosol Reactor. J. Mater. Res. 2000, 15, 733-743.
34. Principles of Environmental Physics; Monteith, J.; Unsworth, M., 1990. 35. Biophysical Ecology; Gates, D. M., 1980.

36. Kulkarni, P.; Biswas, P. A Brownian Dynamics Simulation to Predict Morphology of Nanoparticle Deposits in the Presence of Interparticle Interactions. Aerosol Sci. Technol. 2004, 38, 541-554.

37. Magan, R. V.; Sureshkumar, R. Multiscale-Linking Simulation of Irreversible Colloidal Deposition in the Presence of DLVO Interactions. J. Colloid Interface Sci. 2006, 297, 389-406.

38. Fundamentals of Fluid Mechanics; Munson, B. R.; Young, D. F.; Okiishi, T. H., 1990.

39. Bacher, G.; Szymanski, W. W.; Kaufman, S. L.; Zollner, P.; Blaas, D.; Allmaier, G. Charge-Reduced Nanoelectrospray Ionization Combined with Differential Mobility Analysis of Peptides, Proteins, Glycoproteins, Noncovalent Protein Complexes, and Viruses. J. Mass Spectrom. 2001, 36, 1038-1052.

40. Kaddis, C. S.; Lomeli, S. H.; Yin, S.; Berhane, B.; Apostol, M. I.; Kickhoefer, V. A.; Rome, L. H.; Loo, J. A. Sizing Large Proteins and Protein Complexes by Electrospray Ionization Mass Spectrometry and Ion Mobility. J. Am. Soc. Mass Spectrom. 2007, 18, 1206-1216.

41. Smith, J. N.; Flagan, R. C.; Beauchamp, J. L. Droplet Evaporation and Discharge Dynamics in Electrospray Ionization. J. Phys. Chem. A 2002, 106, 9957-9967.

42. Li, K. Y.; Tu, H. H.; Ray, A. K. Charge Limits on Droplets During Evaporation. Langmuir 2005, 21, 3786-3794

43. Chen, D. R.; Pui, D. Y. H.; Kaufman, S. L. Electrospraying of Conducting Liquids for Monodisperse Aerosol Generation in the $4 \mathrm{~nm}$ to $1.8 \mu \mathrm{m}$ Diameter Range. J. Aerosol Sci. 1995, 26, 963-977.

44. Gomez, A.; Tang, K. Q. Charge and Fission of Droplets in Electrostatic Sprays. Phys. Fluids 1994, 6, 404-414.

45. Tang, X.; Bruce, J. E.; Hill, H. H. Characterizing Electrospray Ionization Using Atmospheric Pressure Ion Mobility Spectrometry. Anal. Chem. 2006, 78, 7751-7760.

46. Scalf, M.; Westphall, M. S.; Smith, L. M. Charge Reduction Electrospray Mass Spectrometry. Anal. Chem. 2000, 72, 52-60.

47. Pan, P.; McLuckey, S. A. Electrospray Ionization of Protein Mixtures a Low pH. Anal. Chem. 2003, 75, 1491-1499.

48. Pan, P.; McLuckey, S. A. The Effect of Small Cations on the Positive Electrospray Responses of Proteins at Low pH. Anal. Chem. 2003, 75, 5468-5474.

49. Nemes, P.; Marginean, I.; Vertes, A. Spraying Mode Effect on Droplet Formation and Ion Chemistry in Electrosprays. Anal. Chem. 2007, 79, 3105-3116. 